\title{
The drag reduction effects of a non-Newtonian fluid (CMC aqueous solution) in a sinusoidal wavy-walled tube at moderate Reynolds numbers for steady flow
}

\author{
YongningBian ${ }^{1, ~ a ~, ~ L i ~ Y u ~}{ }^{2, b}$ and Jing Ren ${ }^{3, c}$ \\ ${ }^{1}$ Da lian University of technology, Liao ning Da lian 116024, China \\ ${ }^{2}$ Da lian University of technology, Liao ning Da lian 116024, China \\ ${ }^{3}$ Da lian University of technology, Liao ning Da lian 116024, China \\ aynbian@dlut.edu.cn.com, ${ }^{\mathrm{b}}$ yuliaixuexi@mail.dlut.edu.cn, ${ }^{\mathrm{c}}$ renjing@mail.dlut.edu.cn
}

\begin{abstract}
Keywords: Drag reduction, CMC aqueous solution, rheological parameters.
Abstract. The drag reduction effects of a non-Newtonian fluid (CMC aqueous solution with 50-1000wppm) in a sinusoidal wavy-walled tube were investigated experimentally in the Reynolds numbers range from 25 to 360 . Based on the pressure drop method, drag reduction was measured and the maximum drag reduction achieved was $38 \%$ using $700 \mathrm{wppm}$. It was found that in the end of the laminar flow region the CMC polymer solutions display more distinct drag reduction than that in turbulent flow region. Besides, the effect of rheological parameters on drag reduction has been discussed.
\end{abstract}

\section{Introduction}

Drag reduction effect has become a hot research topic in the modern fluid mechanics since Toms found the turbulent frictional resistance of polymer solution is less than that of pure solvent in 1948 [1]. The characters of Carbopol, CMC and Glucosesyrup solutions on the heat transfer in turbulent pipe flow were studied and the results indicated that transition to turbulence for non-Newtonian fluids was delayed, the shear thinning and the yield stress enhanced the stability of the flow[2]. The article[3]demonstrated the consistency between the drag reduction behavior and the viscoelastic behavior of polymer solutions and the relationship between viscoelasticity and concentration through viscosity measurements. It was worth noted that CMC solution was not only shear-thinning but also was elastic[4]. In recent years, the mechanism of drag reduction was explained by monitoring the velocity distribution using UDV[5]. However, only a few studies used the non-Newtonian fluids in wavy walled tube which was devoted to the distribution of the flow [1]. The purpose of this work is to investigate the rheology of CMC solution and its effect on the flow in the wavy-walled tube.

\section{Experimental setup and material}

As is shown in Fig.1, the re-circulation flow system used in this experiment is mainly constituted by the following seven parts:1) Receiving tank, 2) Pump, 3 4) Rotameter, 5) Wavy-walled tube, 6) Overflow tank and 7) Heat exchanger. Fig.2 presents the system of pressure drop. The distance between the two measurement points is $332 \mathrm{~mm}$ and there is an one meter flow tube in front of it to ensure the test is done under a fully developed flow region. The wavy-walled tube consists of fourteen wave sections and the diameter at the maximum circular cross section is $10 \mathrm{~mm}$. Thirteen different concentrations of CMC solution are used: (1)low concentration 50-1000wppm, which are mainly used in the experiment of fluid flow in the wavy-walled tube. (2)medium concentration1000-10000wppm, which are mainly used in the rheological test to regress a model. Aqueous solutions of the CMC are prepared by mixing powder into water and stand for $12 \mathrm{~h}$ at room temperature. The rheological characteristics of the tested fluids are determined using the rotational rheometer(Anton paar MCR302)with the concentric cylinder measuring system. 


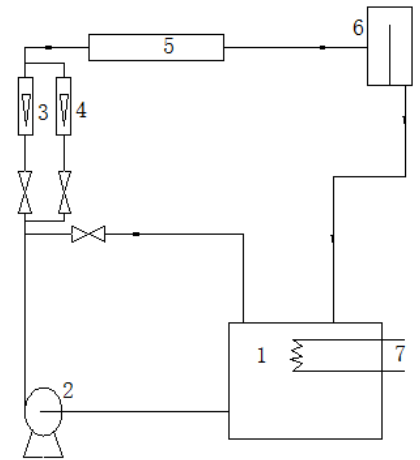

Fig.1 Schematic of the recirculation flow system

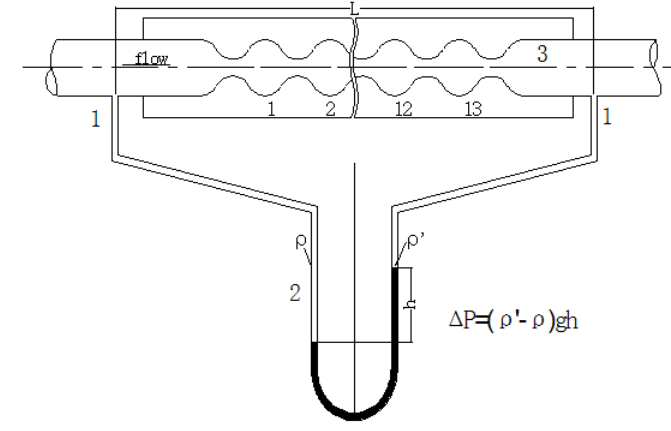

Fig.2 System of pressure drop

The Reynolds number can be simplified as following:

$$
\operatorname{Re}=\frac{\rho u D_{\max }}{\mu}
$$

Where $\rho, u, D_{\max }$ and $\mu$ are fluid density, the tube average velocity, the maximum diameter of the wavy-tube and the viscosity respectively.

The Fanning friction factor can be described as:

$$
f=\frac{\tau_{w}}{\rho u^{2}}=\frac{1}{4} \frac{L}{D_{\max }} \frac{2 \Delta p}{\rho u^{2}}
$$

Where $\tau_{w}$ is the wall shear stress, $L$ is the distance of the two measurement points.

In order to describe the drag reduction of CMC solution, a parameter will be introduced:

$$
\alpha=\frac{\lambda_{w}-\lambda_{c}}{\lambda_{w}}=\frac{\Delta p_{w}-\Delta p_{c}}{\Delta p_{w}}
$$

$\lambda_{w}$ is the friction parameter of water in the tube, $\lambda_{c}$ is the friction parameter of CMC solution, $\Delta P_{w}$ is the pressure drop of water and $\Delta P_{c}$ is the pressure drop of CMC.

\section{Results and discussion}

\section{The rheology of CMC solution with medium and low concentration}

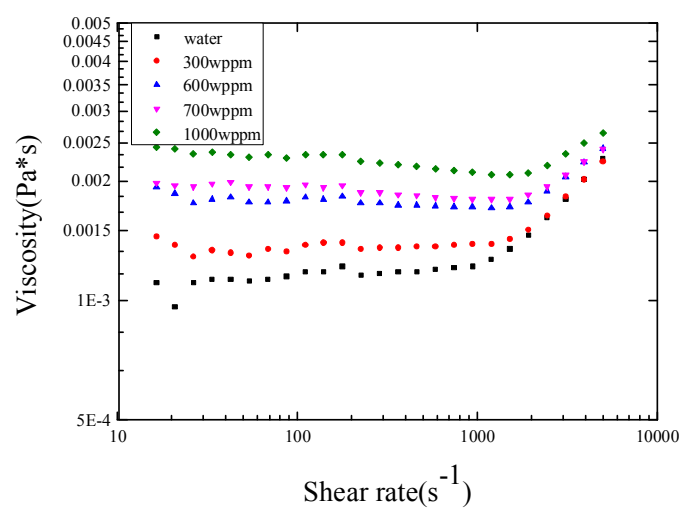

Fig.3 Viscosity curves of CMC solution with low concentration

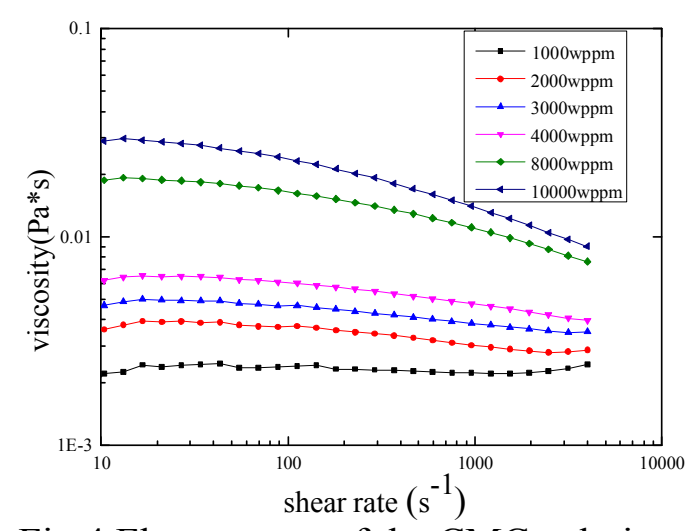

Fig.4 Flow curves of the CMC solution with medium concentration

It is interesting to note, from Fig.3, that in the range of $1<\gamma<1500 s^{-1}$, the viscosity remains usually relatively low and this value hardly depends on the shear rate, so they embody the Newtonian behavior. Another remark is that when the shear rates are high, e.g. at $\&>1500 s^{-1}$, the viscosity begins to increase and part of the reason may be the recoiling and re-entangling of some 
other molecules. Whereas, this phenomenon is hardly to be seen in the case of the medium concentration (see Fig.4). In the range of the medium concentration, we made more than 120 tests to research the effect of temperature $(T)$ and concentration $(C)$ on the consistencies $(a)$ of CMC solutions which were arrived using Ostwald model. On this basis, a equation of the consistencies was deduced: $\ln a=B_{1}+B_{2} * 1000 /(273.15+T)+B_{3} *(\ln C)^{2}+B_{4} \ln C, B_{1}=3.4099, B_{2}=2.0986$ $B_{3}=0.3279, B_{4}=5.0392$. For the low concentration, the parameters achieved using the same method were consistent with the equation, and the relative error was under $5 \%$.

\section{The drag reduction effect of CMC solution in the wavy-walled tube}

Since little effort has been made to the study of the CMC solution in the tube, to our knowledge, the CMC solutions with low concentration are applied in this paper. As discussed above, there is little significant change in the solution viscosity until the shear rate arrives $1500 \mathrm{~s}^{-1}$, and then the effect of shear thinning can be ignored under the fully development pipe flow considerations.

The relationship between the friction factor and the Reynolds number is shown in Fig.5, which is similar to the previous work [1]. For all tested solutions, friction factor decreased with the slope of -1 as the Re increases and the friction factor curve reaches the lowest point. Interestingly, it increases gradually with the Re gets higher, which is almost of independence on the Re. In comparing with the Newtonian fluid, however, it is observed that transitional flow for all CMC solutions is ahead of time. The higher the concentration is, the earlier the transitional regime will be. Besides, the solutions of CMC polymer show a pronounced drag reduction in the laminar flow compared to that in the turbulent flow region. Since the effect of the concentration on drag reduction appears in the form of a lower friction factor at any Re value, it indicates that 700wppm is the optimum drag reduction concentration.

Fig.6 and 7 illustrate the effect of velocity and concentration for CMC solutions on the drag reductions which were obtained using the Eq.(3). They depict that higher drag reduction are achieved when the flow into the end of the laminar flow for all polymer solutions. At the beginning of the laminar flow region, it is hardly to observe an obvious drag reduction effect. To some extent, this is due to the fact that wavy-walled tube can not be able to make the solution disentangle fully at the low velocity. As is expected, drag reduction increases with the increasing of the concentration and drops after the optimum concentration. It is obvious to be seen that $38 \%$ drag reduction exists at the case of $700 \mathrm{wppm}$ solution at the velocity $0.024 \mathrm{~m} / \mathrm{s}$.

We are interested in the effect of rheological parameters such as consistency and flow index on the drag reduction of the $\mathrm{CMC}$ solution. As the tendency of flow index is opposite to the consistency, the two curves are symmetrical. In Fig.8, the consistency increases with the increase of the concentration, the drag reduction reaches its utmost point at the point of 0.0018 , so, the flow index is approximately 0.996 . What can we draw from it is that the closer the fluid index of the polymer approaches to Newtonian fluid, the lesser the drag reduction effect the fluid owns.

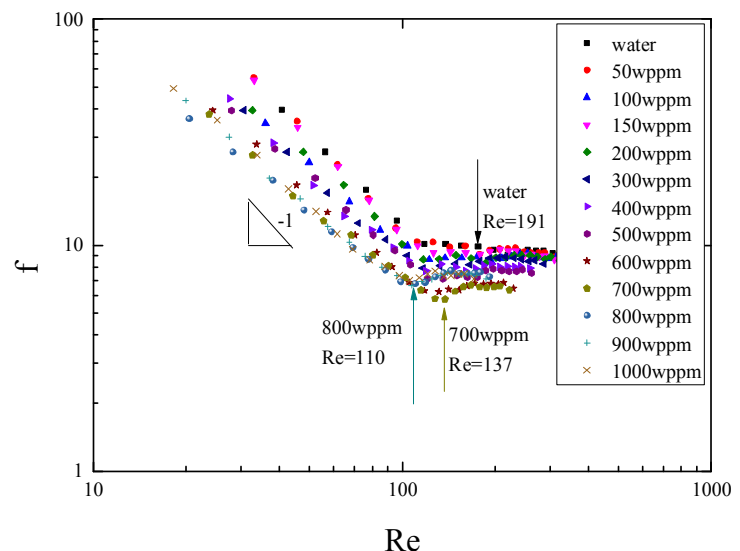

Fig.5 Relationship between friction factor and Re

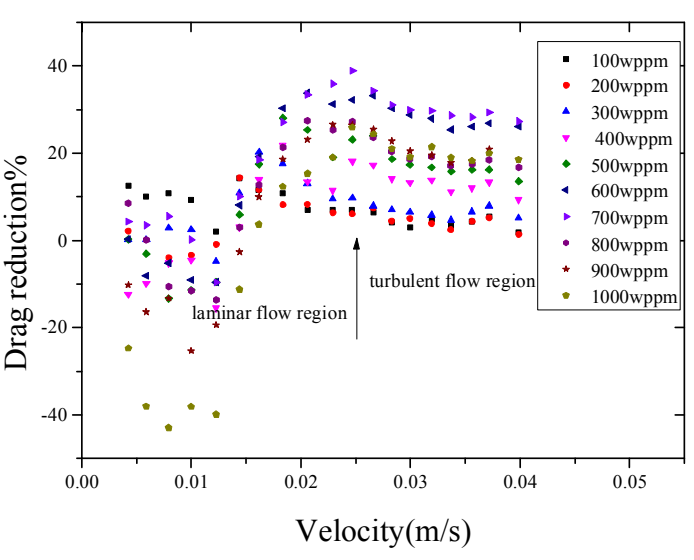

Fig.6 Drag reduction of CMC solution 


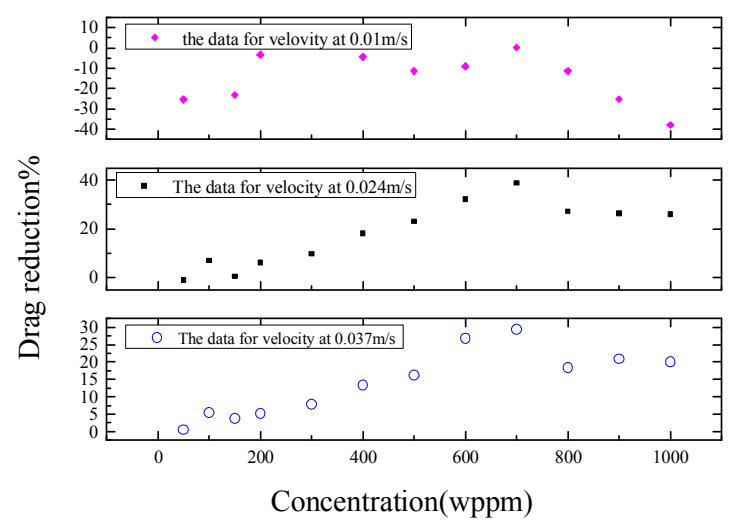

Fig.7 Drag reduction versus polymer concentration at different velocity

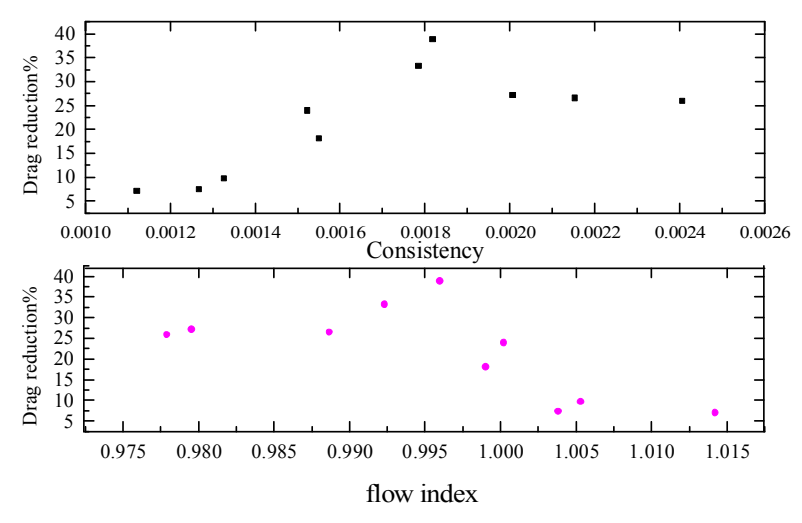

Fig.8 The effect of rheology parameters on the drag reduction

\section{Summary}

The experimental investigation about the drag reduction effect of non-Newtonian fluid with the concentration ranges from 50-1000wppm in a wavy-walled tube for steady flow had been performed. Based on the experimental results, the following conclusions can be reached. Firstly, the distinct drag reduction effect exists in the end of the laminar flow region. The highest drag reduction $38 \%$ can be arrived using $700 \mathrm{wppm}$ which is the optimum concentration solution. Secondly, traditional region is advanced compared to the Newtonian fluid, the flow with higher concentration reaches the traditional region earlier. Thirdly, the closer the fluid index of the polymer to Newtonian fluid reaches, the lesser drag reduction effect the fluid owns. Besides, within the range of experiment, the regression equation of the temperature $(T)$ and concentration $(C)$ on the consistency $(a)$ of CMC solution was $\ln a=B_{1}+B_{2} * 1000 /(273.15+T)+B_{3} *(\ln C)^{2}+B_{4} \ln C$ $B_{1}=3.4099, B_{2}=2.0986, B_{3}=0.3279, B_{4}=5.0392$.

\section{References}

[1]Zonghao Ma,YongningBian:Journal of Experiments in Fluid Mechanics.Vol.24 (2010), p.31

[2]J.Peixinho,C.Desaubry and M.Lebouché:International Journal of Heat and Mass Transfer.Vol.51(2008),p.198

[3]M.P.Escudier, F.Presti and S.Smith:Non-Newtonian Fluid Mech.Vol.81(1999),p.197

[4]A.Fontaine,Y.Guntzburger,F.Bertrand,L.Fradette and M.C.Heuzey:Chemical Engineering Research and Design.Vol.91(2013),p.7

[5]Ş.Zeybek Vural,G.Bayram and Y.Uludag:Turkish Journal of Chemistry.Vol.34(2014),p.142. 